University of South Florida

DIGITAL COMMONS

Digital Commons @ University of

@ UNIVERSITY OF SOUTH FLORIDA

South Florida

8-19-2008

\title{
Estimating Geocenter Variations from a Combination of GRACE and Ocean Model Output
}

\author{
Sean Swenson \\ National Center for Atmospheric Research \\ D. Chambers \\ University of Texas, donc@usf.edu \\ John Wahr \\ University of Colorado
}

Follow this and additional works at: https://digitalcommons.usf.edu/msc_facpub

Part of the Marine Biology Commons

\section{Scholar Commons Citation}

Swenson, Sean; Chambers, D.; and Wahr, John, "Estimating Geocenter Variations from a Combination of GRACE and Ocean Model Output" (2008). Marine Science Faculty Publications. 196.

https://digitalcommons.usf.edu/msc_facpub/196

This Article is brought to you for free and open access by the College of Marine Science at Digital Commons @ University of South Florida. It has been accepted for inclusion in Marine Science Faculty Publications by an authorized administrator of Digital Commons @ University of South Florida. For more information, please contact digitalcommons@usf.edu. 


\title{
Estimating geocenter variations from a combination of GRACE and ocean model output
}

\author{
Sean Swenson, ${ }^{1}$ Don Chambers, ${ }^{2}$ and John $\mathrm{Wahr}^{3}$ \\ Received 10 August 2007; revised 21 February 2008; accepted 7 April 2008; published 19 August 2008. \\ [1] In this study, we estimate a time series of geocenter anomalies from a combination of \\ data from the Gravity Recovery and Climate Experiment (GRACE) satellite mission and the \\ output from ocean models. A matrix equation is derived relating total geocenter variations to \\ the GRACE coefficients of degrees two and higher and to the oceanic component of the \\ degree one coefficients. We estimate the oceanic component from two state-of-the-art ocean \\ models. Results are compared to independent estimates of geocenter derived from other \\ satellite data, such as satellite laser ranging and GPS. Finally, we compute degree one \\ coefficients that are consistent with the processing applied to the GRACE Level-2 gravity \\ field coefficients. The estimated degree one coefficients can be used to improve estimates of \\ mass variability from GRACE, which alone cannot provide them directly.
}

Citation: Swenson, S., D. Chambers, and J. Wahr (2008), Estimating geocenter variations from a combination of GRACE and ocean model output, J. Geophys. Res., 113, B08410, doi:10.1029/2007JB005338.

\section{Introduction}

[2] The Gravity Recovery and Climate Experiment (GRACE) satellite mission, jointly sponsored by NASA and its German counterpart DLR, has been collecting data since mid-2002. The nominal product of the mission is a series of Earth gravity fields, provided in the form of truncated sets of spherical harmonic (Stokes) coefficients at approximately monthly intervals [Tapley et al., 2004a]. Time variations in these coefficients can be used to estimate changes in the distribution of mass within the Earth and at its surface. The recovery of surface mass variability in the form of water, snow, and ice on land and of water in the oceans, is proving to be a particularly useful application of GRACE, e.g., to estimate terrestrial water storage variations from the scale of large river basins [Crowley et al., 2006; Seo et al., 2006] to the continents [Schmidt et al., 2006; Tapley et al., 2004b], for estimating groundwater storage variations [Swenson et al., 2008; Yeh et al., 2006], and for ice sheet and glacier mass loss studies [Velicogna and Wahr, 2006; Tamisiea et al., 2005].

[3] The complete recovery of a surface mass variation requires knowledge of all its spherical harmonic coefficients. To infer mass coefficients from the GRACE Stokes coefficients, it is necessary to separate the Stokes coefficients into contributions from surface mass and contributions from the solid Earth deformation caused by that surface mass. The surface mass contributions are recovered by multiplying the Stokes coefficients by a factor that depends on load Love numbers. This approach does not

\footnotetext{
${ }^{1}$ Advanced Study Program, National Center for Atmospheric Research, Boulder, Colorado, USA.

${ }^{2}$ Center for Space Research, University of Texas, Austin, Texas, USA.

${ }^{3}$ Department of Physics and Cooperative Institute for Research in Environmental Sciences, University of Colorado, Boulder, Colorado, USA.

Copyright 2008 by the American Geophysical Union. 0148-0227/08/2007JB005338\$09.00
}

work for the degree one terms, which are intimately related to the choice of reference frame. For example, the reference frame origin used in the GRACE gravity field determination is the Earth's center of mass (CM). In this frame, the degree one Stokes coefficients from the combined surface mass plus solid Earth are zero by definition, and so no multiplicative factor could allow the recovery of the (presumably) nonzero contribution of the surface mass alone. Thus, until now, there has been no known algorithm for inferring the degree one mass coefficients from the GRACE fields.

[4] The omission of degree one information can have a significant impact on the recovery of high-latitude mass variability and large-scale interbasin ocean mass exchange [e.g., Chen et al., 2005]. For example, Chambers et al. [2004] compared seasonal mean sea level variations from GRACE and steric-corrected Jason-1 altimeter data, and found differences in annual amplitudes on the order of $15 \%$ that were reduced to the $1 \%$ level by the addition of a seasonal degree one estimate. Recently, Chambers et al. [2007] simulated the degree one trends that would arise if ice melted from Greenland, Antarctica, and mountain glaciers at currently observed rates and caused a eustatic sea level rise. They found that ignoring these trends in a GRACE analysis would cause the rate of ocean mass change to be underestimated by up to $30 \%$. In addition, to compare surface loading estimates from GPS to those from GRACE, a degree one correction must be applied [Davis et al., 2004].

[5] Changes in the degree one terms are closely related to motion of the geocenter, defined as the position of the CM relative to the center of figure $(\mathrm{CF})$ of the Earth's outer surface. Previous geocenter estimates based on satellite laser ranging (SLR) have been reported by Chen et al. [1999] and Cretaux et al. [2002]. Chen et al. compared estimates based on Lageos-1 and -2 tracking to estimates based on combined atmosphere, ocean, and hydrological model output. They found general agreement at the annual period, but little 
correlation at the monthly timescale. Cretaux et al. [2002], comparing multiple geocenter and model-predicted solutions, found that the seasonal amplitudes differed by up to a factor of two and the phases differed by as much as 50 days.

[6] Geocenter motion has also been estimated from GPS data [Blewitt and Clarke, 2003], though this method suffers from sampling problems due to the lack of measurements in the oceans and remote land locations such as the tropics [Wu et al., 2002]. To overcome the paucity of data in oceanic regions, an alternate approach was presented by $W u$ et al. [2006], who combined GPS, ocean bottom pressure (OBP) from a data-assimilating model, and GRACE data in a hybrid statistical optimal inversion technique to estimate spectral mass loading coefficients up to degree 50, including degree one. They estimated the uncertainty in their geocenter displacements to be less than $1 \mathrm{~mm}$.

[7] In this study, we use GRACE Stokes coefficients (Release 4 (RL04) data produced by the Center for Space Research (CSR) at the University of Texas) and ocean model output to estimate degree one mass coefficients and geocenter motion. No filters are applied to the data. Degree two zonal coefficients are replaced by values derived from SLR [Cheng and Tapley, 2004]. The technique is rather simple, consisting of a single rank 3 matrix equation relating the global degree one coefficients to their oceanic components. An ocean model is used only to provide the oceanic component of the degree one coefficients, while the oceanic components of higher degree spherical harmonic coefficients are obtained from GRACE. Together these data constrain the global degree one coefficients. The resulting monthly time series of geocenter motions is consistent with previous estimates [Chen et al., 1999; Cretaux et al., 2002; Wu et al., 2006], but has improved accuracy as well as monthly temporal sampling. Moreover, the algorithm described here can be implemented using data that are publicly available as part of the GRACE Project, allowing GRACE users to augment their results by eliminating possible errors due to the omission of degree one gravity field coefficients.

\section{Background}

[8] The Earth's gravitational potential is typically expressed in terms of the geoid, defined as the external equipotential surface that most closely coincides with mean sea level over the ocean. Time variations, $\Delta N$, in the shape of the geoid may be expanded as a sum of spherical harmonic functions

$$
\Delta N(\theta, \phi)=a \sum_{l=0}^{\infty} \sum_{m=0}^{l} \tilde{P}_{l m}(\cos \theta)\left\{\Delta C_{l m}^{\prime} \cos m \phi+\Delta S_{l m}^{\prime} \sin m \phi\right\},
$$

where $\theta$ is colatitude, $\phi$ is longitude, $a$ is the Earth's mean radius, $\tilde{P}_{l m}$ are normalized associated Legendre functions and $\Delta C_{l m}^{\prime}$ and $\Delta S_{l m}^{\prime}$ are time variations in the Stokes coefficients. Using the relationship between the Stokes coefficients and the coefficients describing the corresponding changes in surface mass [Wahr et al., 1998]

$$
\left\{\begin{array}{c}
\Delta C_{l m} \\
\Delta S_{l m}
\end{array}\right\}=\frac{a \rho_{\text {earth }}}{3} \frac{(2 l+1)}{1+k_{l}}\left\{\begin{array}{c}
\Delta C_{l m}^{\prime} \\
\Delta S_{l m}^{\prime}
\end{array}\right\} .
$$

where $\rho_{\text {earth }}$ is the average density of the Earth, and $k_{l}$ are the load Love numbers, one can estimate a field of surface mass anomalies

$$
\Delta \sigma(\theta, \phi)=\sum_{l=0}^{\infty} \sum_{m=0}^{l} \tilde{P}_{l m}(\cos \theta)\left\{\Delta C_{l m} \cos m \phi+\Delta S_{l m} \sin m \phi\right\}
$$

[9] We will refer to the $\Delta C_{l m}$ and $\Delta S_{l m}$ as mass coefficients. In the following, we drop the $\Delta$ notation for clarity, and it should be assumed that these terms represent time variations.

[10] Load Love numbers, $k_{l}$, are used in (2) so that loadinduced deformation within the solid Earth does not contribute to the surface mass estimates. The use of (2) causes all other time-variable solid Earth contributions to be erroneously interpreted as surface mass variability. For the moment, we assume that all these other solid Earth contributions have been modeled and removed from the Stokes coefficients. We will return to this issue below.

\section{Reference Frame Issues}

[11] Equation (2) cannot be used to infer the degree one mass coefficients $\left(C_{10}, C_{11}, S_{11}\right)$ from the GRACE degree one Stokes coefficients $\left(C^{\prime}{ }_{10}, C_{11}^{\prime}, S_{11}^{\prime}\right)$, because those Stokes coefficients vanish in the CM frame used by GRACE. Equation (2) is still accurate in that frame, but the degree one Love number, $k_{1}=-1$ in this frame, so that both the numerator and the denominator in (2) vanish when $l=1$, leaving the degree one mass coefficients undefined. If GRACE were able to recover degree one Stokes coefficients in another frame, one in which those coefficients were not zero and where $k_{1} \neq-1$, then (2) could be used to deliver degree one mass coefficients in that frame.

[12] Estimates of surface mass variability would be most useful if given in the CF frame, because all hydrological/ oceanographic observations and models are made relative to that frame. The $\mathrm{CF}$ and $\mathrm{CM}$ frames are related to one another by a translation. A translation causes a perturbation to the degree one Stokes coefficients [Wu et al., 2002], which is dominated by contributions from the solid Earth, and which can be accounted for by modifying the degree one Love number, $k_{1}$. Specifically, regardless of the frame, (2) shows that the total degree one Stokes coefficients from the surface mass and the deformation it induces in the solid Earth, can be related to the degree one mass coefficients through

$$
\left\{\begin{array}{l}
C_{10}^{\prime} \\
C_{11}^{\prime} \\
S_{11}^{\prime 1}
\end{array}\right\}=\frac{1+k_{1}}{a \rho_{\text {earth }}}\left\{\begin{array}{l}
C_{10} \\
C_{11} \\
S_{11}
\end{array}\right\}
$$

[13] Different frames are characterized by different values of $k_{1}$. In the CM frame, $k_{1}=-1$, so that the Stokes coefficients vanish. In the CF frame, $k_{1}=0.021$ [Blewitt, 2003; Trupin et al., 1992]. In this paper, we will describe a method of finding the degree one mass coefficients, $\left(C_{10}\right.$, $\left.C_{11}, S_{11}\right)$, using the GRACE Stokes coefficients at degrees $l \geq 2$. Once we have found those mass coefficients, we will 
construct degree one Stokes coefficients using (4) with $k_{1}=$ 0.021: i.e., in the CF frame.

[14] The construction of those degree one Stokes coefficients is the primary goal of this paper. We will also use those coefficients to estimate geocenter motion, and will compare our geocenter results with previously published studies. Such studies typically use the notation $(X, Y, Z)$ to denote the Cartesian coordinates of the geocenter in the terrestrial reference frame [Cretaux et al., 2002]. Those coordinates are related to the degree one Stokes coefficients in the CF frame, by Cretaux et al. [2002]:

$$
\begin{aligned}
& X=\sqrt{3} a C_{11}^{\prime} \\
& Y=\sqrt{3} a S_{11}^{\prime} \\
& Z=\sqrt{3} a C_{10}^{\prime},
\end{aligned}
$$

\section{Methods}

\subsection{Oceanic Component of Geocenter}

[15] If we partition the surface mass signal into land and ocean components, equation (3) may be written as
[17] Equation (9) shows that each oceanic spherical harmonic coefficient, $C_{l m}^{\text {ocean }}$, has a contribution from each and every global coefficient, $\left(C_{l m}, S_{l m}\right)$. A similar result holds for $S_{l m}^{\text {ocean }}$. Evaluating (9) for $(l, m)=(1,0)$ and separating out the $(1,0)$ term on the right-hand side, gives:

$$
\begin{aligned}
C_{10}^{\text {ocean }}= & \frac{C_{10}}{4 \pi} \int d \Omega \tilde{P}_{10}(\cos \theta) \vartheta(\theta, \phi) \tilde{P}_{10}(\cos \theta) \\
& +\frac{1}{4 \pi} \int d \Omega \tilde{P}_{10}(\cos \theta) \vartheta(\theta, \phi) \\
& \cdot \sum_{l=0}^{\infty} \sum_{m=0}^{l} \tilde{P}_{l m}(\cos \theta)\left\{C_{l m} \cos m \phi+S_{l m} \sin m \phi\right\},
\end{aligned}
$$

where the summations are understood to exclude the $(1,0)$ term.

[18] Now assume that (1) all the original mass coefficients are known except for $C_{10}$, and (2) $C_{10}^{\text {ocean }}$ is known (e.g., from an ocean model). Under these conditions, 9 is one equation with one unknown $\left(C_{10}\right)$, and we can rearrange that equation to obtain an expression for $C_{10}$ in terms of the known oceanic component and the remaining original mass coefficients:

$$
C_{10}=\frac{4 \pi C_{10}^{\text {ocean }}-\int d \Omega \tilde{P}_{10}(\cos \theta) \vartheta(\theta, \phi) \sum_{l=0}^{\infty} \sum_{m=0}^{l} \tilde{P}_{l m}(\cos \theta)\left\{C_{l m} \cos m \phi+S_{l m} \sin m \phi\right\}}{\int d \Omega \tilde{P}_{10}(\cos \theta) \vartheta(\theta, \phi) \tilde{P}_{10}(\cos \theta)},
$$

$$
\begin{aligned}
\sigma(\theta, \phi) & =L(\theta, \phi) \sigma(\theta, \phi)+\vartheta(\theta, \phi) \sigma(\theta, \phi) \\
& =\sigma^{\text {land }}(\theta, \phi)+\sigma^{\text {ocean }}(\theta, \phi) \\
& =\sum_{l=0}^{\infty} \sum_{m=0}^{l} \tilde{P}_{l m}(\cos \theta)\left\{\left(C_{l m}^{\text {land }}+C_{l m}^{\text {ocean }}\right) \cos m \phi\right. \\
& \left.+\left(S_{l m}^{\text {land }}+S_{l m}^{\text {ocean }}\right) \sin m \phi\right\},
\end{aligned}
$$

where $L$ represents the land function

$$
L(\theta, \phi)=\begin{gathered}
1 \text { if land } \\
0 \text { if ocean }
\end{gathered},
$$

and $\vartheta=1-L$ represents the corresponding ocean function. Here $C_{l m}^{\text {ocean }}$ and $S_{l m}^{\text {ocean }}$ are the mass coefficients of $\sigma^{\text {ocean }}=$ $\vartheta \sigma$, the ocean component of $\sigma$.

[16] The orthogonality of the $\tilde{P}_{l m}$ 's over the sphere can be used to obtain

$$
C_{l m}^{\text {ocean }}=\frac{1}{4 \pi} \int d \Omega \tilde{P}_{l m}(\cos \theta) \cos m \phi \vartheta(\theta, \phi) \sigma(\theta, \phi),
$$

and similarly for $S_{l m}^{\text {ocean }}$. The coefficients describing $\sigma^{\text {ocean }}$ can be expressed in terms of the original mass coefficients using equation (3) for $\sigma$ in equation (8)

$$
\begin{aligned}
C_{l m}^{\text {ocean }}= & \frac{1}{4 \pi} \int d \Omega \tilde{P}_{l m}(\cos \theta) \cos m \phi \vartheta(\theta, \phi) \\
& \cdot \sum_{l^{\prime}=0}^{\infty} \sum_{m^{\prime}=0}^{l^{\prime}} \cdot \tilde{P}_{l^{\prime} m^{\prime}}(\cos \theta)\left\{C_{l^{\prime} m^{\prime}} \cos m^{\prime} \phi+S_{l^{\prime} m^{\prime}} \sin m^{\prime} \phi\right\} .
\end{aligned}
$$

[19] Equation (11) can be used to find $C_{10}$, given the values of the other mass coefficients and of $C_{10}^{\text {ocean }}$.

[20] Equation (10) can be extended to the case of an arbitrary number of unknown coefficients (with the same number of known oceanic components). For example, the specific case where all degree one terms are unknown, (e.g., GRACE), becomes

$$
\left[\begin{array}{l}
C_{10}^{\text {ocean }} \\
C_{11}^{\text {Cocean }} \\
S_{11}^{\text {ocean }}
\end{array}\right]=\left[\begin{array}{lll}
I_{10 C}^{10 C} & I_{11 C}^{10 C} & I_{11 S}^{10 C} \\
I_{10 C}^{11 C} & I_{11 C}^{11 C} & I_{11 S}^{11 C} \\
I_{10 C}^{11 S} & I_{11 C}^{11 S} & I_{11 S}^{11 S}
\end{array}\right]\left[\begin{array}{l}
C_{10} \\
C_{11} \\
S_{11}
\end{array}\right]+\left[\begin{array}{l}
G_{10 C} \\
G_{11 C} \\
G_{11 S}
\end{array}\right]
$$

where we have used the notation

$$
I_{10 C}^{10 C}=\frac{1}{4 \pi} \int d \Omega \tilde{P}_{10}(\cos \theta) \vartheta(\theta, \phi) \tilde{P}_{10}(\cos \theta),
$$

the superscript indicating the spherical harmonic to the left of $\vartheta$ and the subscript indicating that to the right, and

$$
\begin{aligned}
G_{10 C}= & \frac{1}{4 \pi} \int d \Omega \tilde{P}_{10}(\cos \theta) \vartheta(\theta, \phi) \\
& \cdot \sum_{l=0}^{\infty} \sum_{m=0}^{l} \cdot \tilde{P}_{l m}(\cos \theta)\left\{C_{l m} \cos m \phi+S_{l m} \sin m \phi\right\} .
\end{aligned}
$$

[21] Here the summations exclude all degree one terms. Equation (12) can be used to solve for $\left(C_{10}, C_{11}, S_{11}\right)$, given knowledge of the oceanic contributions and of each $\left(C_{l m}\right.$, $\left.S_{l m}\right)$ for $l \geq 2$. The degree one Stokes coefficients in the CF 
frame can then be found using (4) with $k_{1}=0.021$, and geocenter coordinates can be computed using (5).

[22] While equation (12) is formulated solely in terms of oceanic contributions (via $\vartheta$ ), mass variations over land are accounted for implicitly. This can be seen by replacing $\vartheta$ by $(1-L)$ in equation (14). The orthogonality of the $P_{l m}$ results in an equation in which $\vartheta$ is replaced by $-L$, showing that the $G$ vector is equal to the negative of the contribution from mass variations on land. Moving these terms to the left-hand side of (12) results in an equivalent equation with an interpretation that is perhaps more intuitive to some readers: GRACE is used to infer mass variations over land, from which the land component of the degree one terms are estimated. To these values, an ocean component is added. The $I$ matrix accounts for the fact that the GRACE data only included degrees 2 and higher. It can be shown that the $I$ matrix is mathematically equivalent to an iterative procedure, whereby the initial degree one coefficients $\left(C_{10}^{0}\right.$, $C_{11}^{0}, S_{11}^{0}$ ) obtained by adding the GRACE derived land components to the model derived ocean components, i.e.,

$$
\left[\begin{array}{l}
C_{10}^{\text {ocean }} \\
C_{11}^{\text {ocean }} \\
S_{11}^{\text {ocean }}
\end{array}\right]-\left[\begin{array}{l}
G_{10 C} \\
G_{11 C} \\
G_{11 S}
\end{array}\right]=\left[\begin{array}{l}
C_{10}^{0} \\
C_{11}^{0} \\
S_{11}^{0}
\end{array}\right]
$$

are used to update $\left(G_{10 C}, G_{11 C}, G_{11 S}\right)$ until $\left(C_{10}, C_{11}, S_{11}\right)$ converge.

\subsection{GRACE Processing Standard}

[23] The mass coefficients discussed in the previous section describe mass variations of the atmosphere, oceans, and terrestrial water storage. As part of the processing performed by the GRACE Project, the GRACE Stokes coefficients (denoted by the GRACE Project as "GSM" coefficients) have had modeled estimates of the atmospheric and oceanic mass signals removed. Thus the GRACE coefficients include the full effects of terrestrial water storage, but the only remaining atmospheric and oceanic signals are those due to errors in the respective models. The GRACE Project provides the modeled atmospheric and oceanic contributions to the Stokes coefficients in two forms: "GAC" files which include the global atmospheric and oceanic effects, and "GAD" files which have had the atmospheric signals over land set to zero. The coefficients in the GAD file therefore represent ocean bottom pressure variations.

[24] Below, we compute results for two types of related quantities. One is the set of degree one Stokes coefficients $\left(C_{10}^{\prime}, C_{11}^{\prime}, S_{11}^{\prime}\right)$ that are most compatible with the GSM fields. These coefficients have had atmospheric and oceanic contributions removed, using the same atmospheric and oceanic models used to construct the GSM fields. We will refer to these as "GSM-like" results. The other is the set of geocenter coordinates $(X, Y, Z)$ that contain all geophysical contributions, including those from the entire atmosphere and ocean, as well as from terrestrial water storage and the solid Earth. These are useful as stand-alone quantities and can be directly compared with SLR and GPS estimates. We will refer to these as "full-geocenter" results.

[25] When using (12) to solve for $\left(C_{10}, C_{11}, S_{11}\right)$, the values used for $\left(C_{10}^{\text {ocean }}, C_{11}^{\text {ocean }}, S_{11}^{\text {ocean }}\right)$ must be compatible with those used for the $\left(C_{l m}, S_{l m}\right)$. There are two possible ways of accomplishing this:

[26] 1. The GRACE GSM coefficients can be used as given, for $l \geq 2$. Because atmospheric and oceanic model output has been removed from those coefficients, output from those same models should then be removed from the estimates of $\left(C_{10}^{\text {ocean }}, C_{11}^{\text {ocean }}, S_{11}^{\text {ocean }}\right)$, i.e.,

$$
\left[\begin{array}{l}
C_{10}^{\text {ocean }} \\
C_{11}^{\text {ocean }} \\
S_{11}^{\text {ocean }}
\end{array}\right] \Rightarrow\left[\begin{array}{l}
C_{10}^{\text {ocean }}-G A D_{10 C} \\
C_{11}^{\text {ocean }}-G A D_{11 C} \\
S_{11}^{\text {ocean }}-G A D_{11 S}
\end{array}\right]
$$

where $G A D_{10 C}$, etc. refer to degree one mass coefficients for the same model of ocean bottom pressure used to generate the GAD Stokes coefficients. The Stokes coefficients of this model, including degree one, are routinely provided by the GRACE Project. Because the GRACE Project uses $k_{1}=0$ when computing the degree one GAD Stokes coefficients, $k_{1}=0$ must be used to convert these coefficients back to mass units for use in (16). The solutions for $\left(C_{10}^{\prime}, C_{11}^{\prime}, S_{11}^{\prime}\right)$ obtained by solving (12) and then using the results in (4) with $k_{1}=0.021$, are estimates of GSM-like Stokes coefficients in the CF frame. Full geocenter results - ones that include all atmospheric and oceanic signals - can be obtained from these GSM-like results by adding the degree one Stokes coefficients for the GAC model (thus adding back the contributions from the atmospheric and oceanic models), and then using the resulting total degree one Stokes coefficients in (5). The GRACE Project also assumes that $k_{1}=0$ when computing the degree one GAC Stokes coefficients, so to generate consistent geocenter coordinates, the degree one GAC estimates provided by the GRACE Project should be multiplied by 1.021 before being added to the full geocenter results.

[27] 2. Alternatively, the modeled atmospheric and oceanic contributions could be restored to the $l \geq 2 \mathrm{GSM}$ coefficients before computing $G(14)$, i.e.,

$$
\left[\begin{array}{c}
C_{l m} \\
S_{l m}
\end{array}\right] \Rightarrow\left[\begin{array}{c}
C_{l m}+C_{10 C}^{G A D} \\
S_{l m}+S_{11 C}^{G A D}
\end{array}\right]
$$

[28] In that case the values used for $\left(C_{10}^{\text {ocean }}, C_{11}^{\text {ocean }}, S_{11}^{\text {ocean }}\right)$ should include the total contribution from ocean bottom pressure, not the departure from the GAD model (17). The solution of (12) would then be used in (4) with $k_{1}=0.021$, to give the degree one Stokes coefficients in the CF frame with the entire atmosphere and ocean included. These could then be converted into full geocenter coordinates using (5), or the degree one GAC Stokes coefficients (obtained by multiplying the Project-provided degree one GAC coefficients by 1.021, as described above) could be subtracted from the total degree one Stokes coefficients to obtain GSM-like results.

[29] It can be shown that these two methods give identical results, provided the sum over $l$ in (14) goes to infinity. In practice, there are small differences because that sum is truncated: CSR RL04 monthly coefficients are provided only up through degree $l_{\max }=60$, for example. Those differences are small enough, however, that either method is adequate. 


\subsection{Post-Glacial Rebound}

[30] The GRACE GSM coefficients include contributions from the solid Earth. Thus both the full-geocenter and the GSM-like results should include solid Earth contributions. The derivation described in section 4.1 assumes the Stokes coefficients include no solid Earth contributions. When using the $l \geq 2$ GSM coefficients to compute the $G$ vector on the right-hand side of (12), solid Earth contributions therefore should first be modeled and removed. Then GSMlike and full geocenter solutions should be found as described above, and degree one solid Earth coefficients should be used to add the solid Earth effects back to those solutions.

[31] The only time-variable solid Earth contributions that have been detected in GRACE measurements (other than those caused by surface loading, which are already fully accommodated by $k_{l}$ in (2)) are post-glacial rebound [Tamisiea et al., 2007; Paulson et al., 2007] and the 2004 Sumatran earthquake [Han et al., 2006]. Here we do not attempt to model the effects of the Sumatran earthquake. For post-glacial rebound we remove the $l \geq 2$ model results of Paulson et al. [2007], which are computed using the global ICE-5G ice deglaciation model and viscosity profile VM2 (both described by Peltier [2004]). The degree one coefficients from post-glacial rebound are small enough that they do not need to be added to either the GSM-like or full geocenter solutions. Their contributions to the geocenter coordinates, for example, are on the order of $0.1 \mathrm{~mm} / \mathrm{yr}$, which is far smaller than the numerical results presented below [see also Argus et al., 1999].

\section{Ocean Models}

[32] A formulation similar to (12) could be derived by assuming the degree-one land components, rather than the ocean components, are known. However, land mass variations, which are significantly larger than oceanic variations, are arguably less well modeled and include such poorly understood regions as Greenland and Antarctica. Ocean mass variations, in contrast, consist of a nearly uniform eustatic signal that can be measured with GRACE [e.g., Chambers et al., 2004], as well as more localized variations associated with mass redistribution within the ocean that can be modeled with reasonable accuracy [e.g., Fukumori et al., 1999].

[33] Changes in gravity on land, such as from melting of ice sheets and variations in water storage, will cause the ocean mass to depart from eustacy slightly. Such changes are not included in ocean models. Several studies [e.g., Tamisiea et al., 2001] have estimated the size of this departure based on models of ice sheet melting and the solid Earth. They find that the self-gravitational changes can cause sea level to depart from a eustatic change by up to $\pm 30 \%$ when only one ice sheet is considered. The patterns are long-wavelength, and the higher sea level change is far from the source of the melting, while the lower sea level is closer. Since Greenland, Antarctica, and mountain glaciers are all in difference hemispheres, this means that the patterns tend to cancel slightly so that the overall departure from eustacy is closer to $\pm 10 \%$ in the deep ocean. However, these studies focus only on the small $\frac{\mathrm{mm}}{\mathrm{yr}}$ secular change in sea level and ignore the much larger $(16 \mathrm{~mm}$ changes over the period of 1 year) that is not from ice sheet melting, but is from the exchange of water between the land and ocean as part of the seasonal water cycle [e.g., Chambers et al., 2004]. We are not aware of any study that has derived seasonal self-gravitation patterns of sea level rise related to land water storage change and we did not want to include patterns of ice sheet melting effects [e.g., Tamisiea et al., 2001] without including patterns of self-gravitation from hydrology changes. Therefore we add a uniform ocean mass fluctuation to the ocean models and ignore the currently uncertain self-gravitational signals. We note that the eustatic correction we apply is nonetheless likely to be a significant improvement over an assumption of constant ocean mass.

[34] To model the ocean bottom pressure (OBP) variations associated with mass redistribution signals in the ocean (i.e., the left-hand side of equation (12)), we rely on the output of two numerical ocean models. The first is a version of the MIT general circulation model [Marshall et $a l ., 1997]$ that is run at Jet Propulsion Laboratory (JPL) as part of the Estimating the Circulation and Climate of the Ocean (ECCO) consortium. This is a baroclinic model forced by winds, pressure, and heat and freshwater fluxes from the National Center for Environmental Prediction (NCEP) operational analyses products [Fukumori et al., 1999]. We use the simulation version that does not assimilate altimetry data because it extends over a longer time period. However, we compared the OBP from both the simulation and data assimilating models over coincident months (2002 to 2005) and found they agreed well for the longest wavelengths that are important for determining the geocenter. The JPL ECCO model extends only between $\pm 78^{\circ}$ latitude, so it does not model barotropic fluctuations in the Arctic Ocean or near Antarctica.

[35] The second model is the Ocean Model for Circulation and Tides (OMCT), used by the GRACE Project to compute high-frequency ocean bottom pressure (OBP) in order to de-alias GRACE data during processing [Thomas, 2002; Flechtner, 2007]. The monthly averaged OBP from this model is distributed along with the GRACE gravity coefficients, both separately (in the GRACE GAD files) and combined with the atmospheric mass signal over land (in the GRACE GAC files). Similar to ECCO, this is a baroclinic model forced by winds, pressure, and heat and freshwater fluxes from the ECMWF operational analyses. In contrast to ECCO, OMCT models the entire ocean, including the Arctic and Southern Oceans.

[36] Because ECCO and OMCT are not coupled oceanatmosphere-land models, the eustatic fluctuation in OBP caused by the exchange of water mass among these components is not represented in their OBP estimates. This eustatic signal is presumably present in the GRACE GSM coefficients used to compute $G$ in (12). Thus the eustatic signal needs to be included in $\left(C_{10}^{\text {ocean }}, C_{11}^{\text {ocean }}, S_{11}^{\text {ocean }}\right)$ when solving (12). To model the eustatic component of global mean sea level, the monthly transport of water between land and ocean is derived from GRACE measurements [Chambers et al., 2004]. We use GRACE coefficients from CSR RL04 from August 2002 to December 2006, where the $C_{20}^{\prime}$ coefficients have been replaced with values from an analysis of satellite laser ranging (SLR) data [Cheng and Tapley, 2004], and an initial estimate of the mean seasonal geocenter variability [Chambers et al., 2004] and a model 

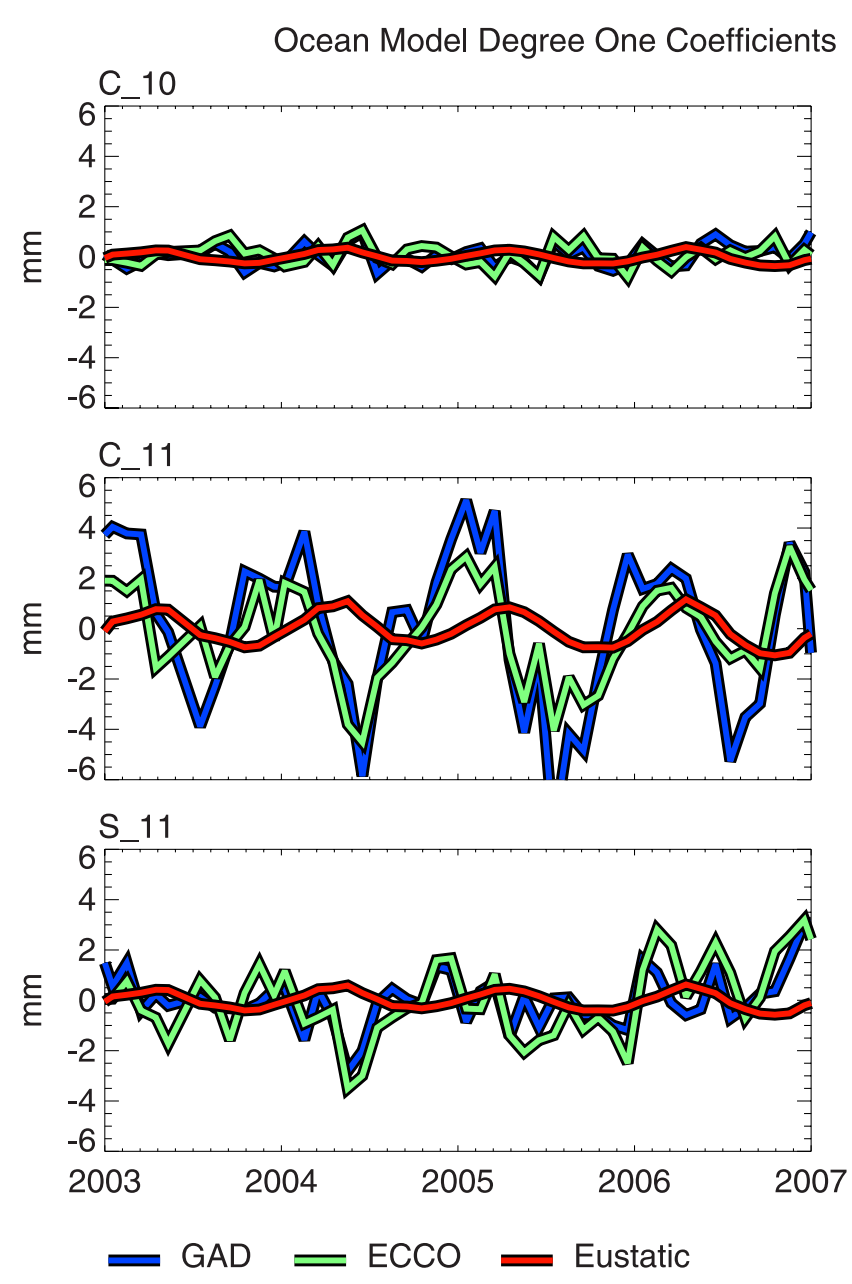

Figure 1. Time series of oceanic component of degree one coefficients. The $y$ axis indicates geocenter in millimeters; the $x$ axis is time in years. Blue line represents values derived from OMCT ocean model, green line represents values derived from ECCO ocean model, and red line represents values derived from eustatic correction. (top) $C_{10}^{\text {ocean }}$. (middle) $C_{11}^{\text {ocean }}$. (bottom) $S_{11}^{\text {ocean }}$.

for post-glacial rebound [Paulson et al., 2007] have been applied.

[37] Figure 1 shows the time series of the degree one coefficients estimated from these models, presented here and in all later figures in terms of geocenter displacements (5). Figure 1 (top) shows $C_{10}^{\text {ocean }}$, Figure 1 (middle) shows $C_{11}^{\text {ocean }}$, and Figure 1 (bottom) shows $S_{11}^{\text {ocean }}$. In each figure, the coefficients are plotted in $\mathrm{mm}$ of geocenter motion, and the three lines represent the ECCO and OMCT ocean models, as well as the eustatic correction. All three coefficients estimated from the eustatic ocean model show a clear seasonal cycle having an amplitude of between 0.5 and $1 \mathrm{~mm}$. The degree one terms derived from the two numerical models show behavior similar to one another. A relatively large, though noisy, seasonal cycle can be seen for $C_{11}^{\text {ocean }}$, but not for $C_{10}^{\text {ocean }}$ and $S_{11}^{\text {ocean }}$. Furthermore, the amplitude of $C_{11}^{\text {ocean }}$ is significantly larger than that of either $C_{10}^{\text {ocean }}$ or $S_{11}^{\text {ocean }}$; this difference reflects a seasonal redistribution of water between the ocean basins. To isolate the influence of the unmodeled polar regions in ECCO, degree one coefficients were com- puted from OMCT using only points between $\pm 78^{\circ}$ latitude; these coefficients differed negligibly from the original, indicating that noneustatic mass variability in the Arctic Ocean is not great enough to significantly affect the degree one coefficients.

\section{Results}

\subsection{Synthetic Experiment}

[38] To confirm that the methodology can recover global degree one coefficients, we first apply it to a set of synthetic coefficients constructed from a combined land-ocean model. Gridded output of the Noah land surface model, driven with forcing fields from the Global Land Data Assimilation System (GLDAS) [Rodell et al., 2004], is combined with a modeled estimate of ocean mass variations. In this example, we use the spatially uniform, eustatic ocean model previously described, although we note that the method works equally well for other ocean models. The combined, gridded model output is converted to spherical harmonic coefficients and truncated to some maximum degree, $l_{\max }$. The choice $l_{\max }=60$ is consistent with the CSR RL04 fields. The degree one terms are saved for later comparison with the recovered terms, then set to zero. Figure 2 shows the results of this experiment for synthetic coefficient sets that use three choices of $l_{\max }: 179,60$, and 5 . All results are expressed in terms of geocenter coordinates, computed as described at the end of section 4.1. For each coefficient, the black line represents the original degree one term. The rms differences for each $l_{\max }$ are approximately 4,10 , and $50 \%$ of the original degree one term. Thus we expect that in the absence of errors in either the ocean model degree one term or the GRACE degree two and higher coefficients, the monthly geocenter coefficients should be recoverable to within $10 \%$.

\subsection{Full Geocenter Estimates}

[39] Having demonstrated the viability of the method on synthetic data, we now estimate full geocenter coefficients from actual GRACE data. Figure 3 shows the results of using each ocean model, after adding the eustatic correction, with CSR RL04 GRACE data in equation (12). The blue and red lines represent the full geocenter time series based on the ECCO and OMCT ocean models respectively. Two independent full geocenter estimates are also plotted: the SLR-based estimate of Eanes [2000] (orange line) and the GPS hybrid estimate of $W u$ et al. [2006] (black line). The Eanes results are not monthly values, nor do they extend into the GRACE period of record. Rather, the time series describes the mean annual cycle for the period 1992 2000. The Wu et al. [2002] monthly time series extends into the current GRACE period, but ends in mid-2004. In addition, $\mathrm{Wu}$ et al. removed a long-term trend from their results. For consistency, from each time series we have removed the mean over the time period of the shortest record (that of the $W u$ et al. [2002] estimates).

[40] All the estimates show a clear annual cycle, and similar amplitudes. The $Z$ and $X$ coefficients are relatively close in phase, with a maximum occurring in boreal spring, while the $Y$ coefficient peaks in winter. An examination of the relative contributions of the GSM and GAC coefficients to $G$ in equation (12) reveals that much of the month-tomonth variability derives from the GAC ocean/atmosphere 


\section{Synthetic Degree One Recovery}
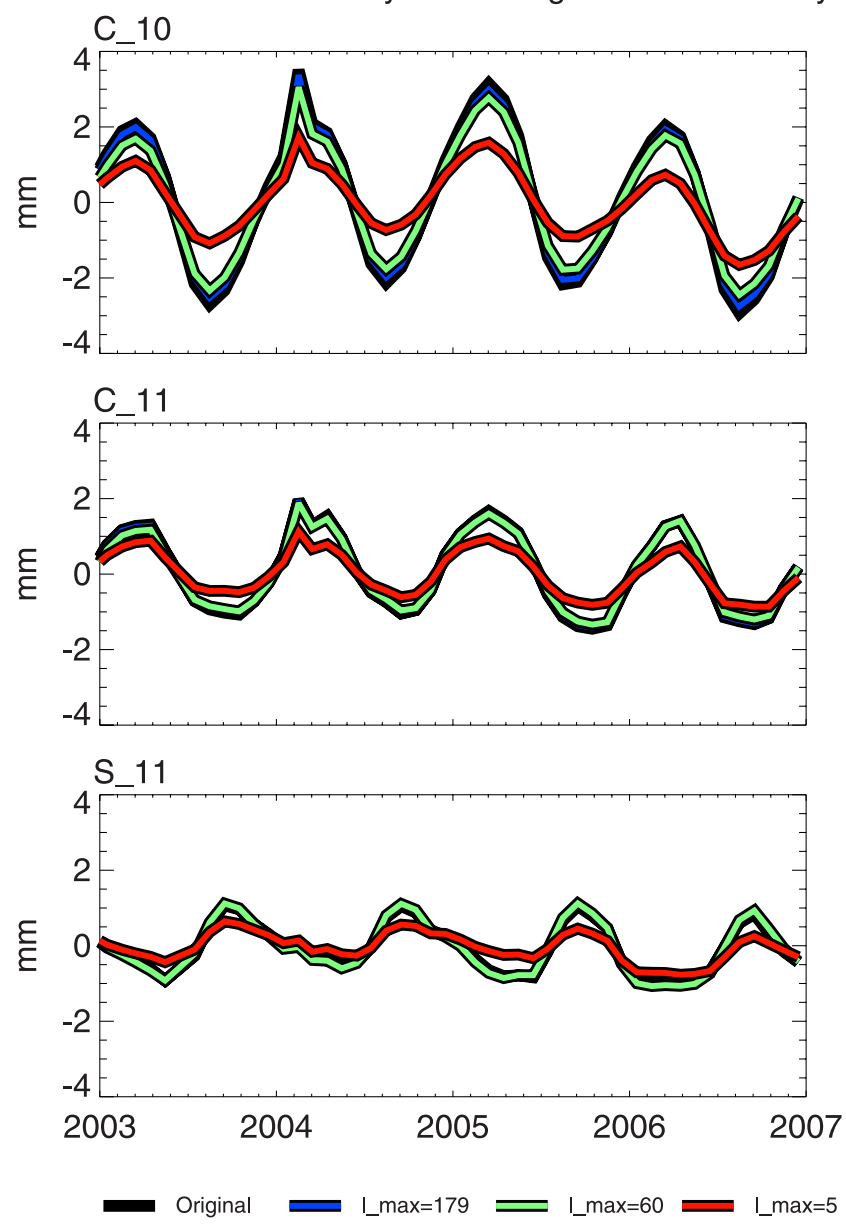

Figure 2. Time series of recovered monthly geocenter coordinates derived from synthetic, model-based Stokes coefficients. Black line represents original results. Blue, green, and red, lines are derived from equation (12) truncated to degrees 179,60 , and 5 respectively. (top) $Z$. (middle) $X$. (bottom) $Y$. The $y$ axis is in millimeters. The $x$ axis is time in years.

coefficients, especially for $Z$. To focus on the seasonal cycle, a low-pass filter (consisting of the annual cycle resulting from a moving, Gaussian weighted least squares fit) is applied to each time series. Figure 4 shows the comparison after low-pass filtering the $\mathrm{GRACE}^{E C C O}$, GRACE $^{O M C T}$, and $W u$ et al. [2002] time series.

[41] The $Z$ coefficients of the Wu et al. [2002] and Eanes time series agree closely in phase, but the $\mathrm{Wu}$ et al. amplitude is about 1.5 times greater. The amplitudes of the GRACE ${ }^{E C C O}$ and GRACE ${ }^{O M C T}$ time series are about $50 \%$ and $80 \%$ of Eanes, while the phases are delayed by two to three weeks. The $X$ coefficients have similar phases, differing by one week at most. The GRACE ${ }^{O M C T}$ amplitude is about $10 \%$ smaller than Eanes. In contrast, GRACE ${ }^{E C C O}$ and $W u$ et al. [2002] $X$ values are smaller during the time period shown. $Y$ shows close agreement in phase between the various degree one estimates in Figure 4. The Wu et al. time series has a slightly smaller amplitude, while the other three estimates are nearly the same.
[42] The mean annual cycle of each time series is listed in Table 1, along with previously published values. Annual amplitudes derived from the OMCT model are greater than those derived from ECCO for $X$ and $Z$, and approximately the same for $Y$. GRACE ${ }^{E C C O}$ and GRACE ${ }^{O M C T}$ annual phases are within about one week. Earlier estimates reported by Chen et al. [1999], Eanes [2000], Cretaux et al. [2002], and $W u$ et al. [2006] are all of greater amplitude than our estimates. Mean annual phases are within about one month for all components, with the largest spread of values for $Z$.

[43] The largest difference between the GRACE ${ }^{E C C O}$ and GRACE $^{O M C T}$ time series can be seen in $X$. This may be due to the difference in the annual amplitudes of the ECCO and OMCT models; the amplitude ratio of OMCT to ECCO is 2.1. GRACE ${ }^{E C C O}$ and GRACE ${ }^{O M C T}$ differ most notably from the SLR and GPS based estimates for $Z$, which may be related to the sampling bias present in the SLR and GPS

\section{C_10}

CSR RL04
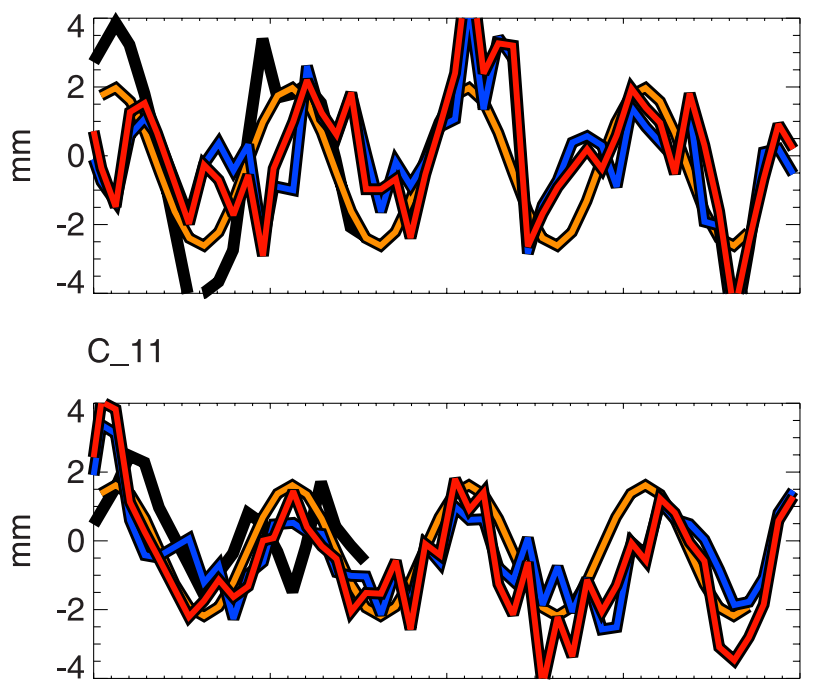

\section{S_11}

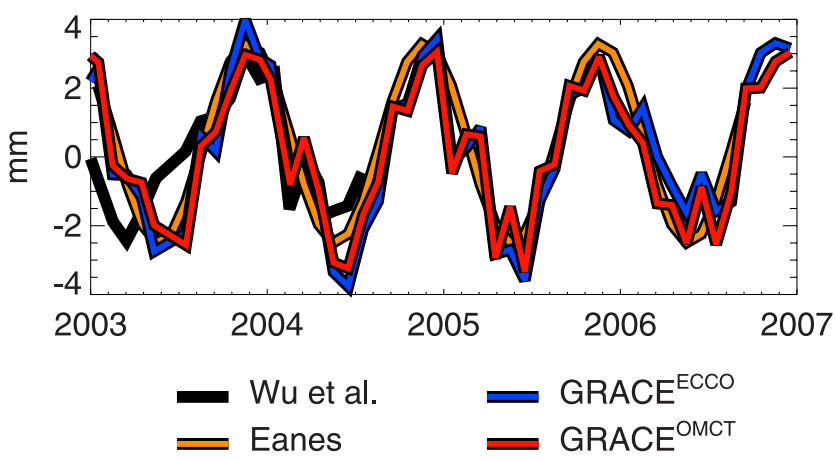

Figure 3. Time series of full geocenter estimates computed using GRACE Stokes coefficients and degree one ocean model results. Blue and red lines are derived from equation (12) using ECCO and OMCT ocean models, respectively, after adding a eustatic correction to each model. Black line represents values estimated by Wu et al. [2006], and orange line represents mean seasonal estimate from Eanes [2000]. (top) Z. (middle) $X$. (bottom) $Y$. The $y$ axis is in millimeters. The $x$ axis is time in years. 
C_10

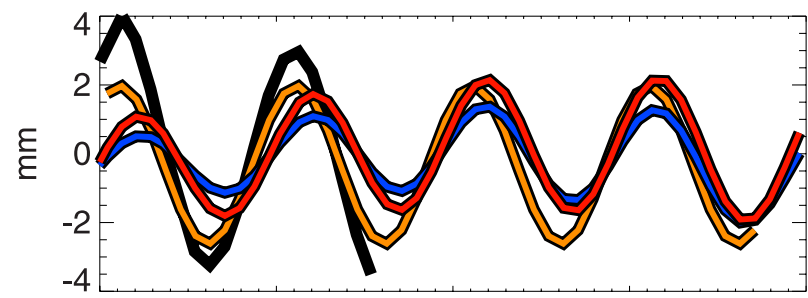

C_11

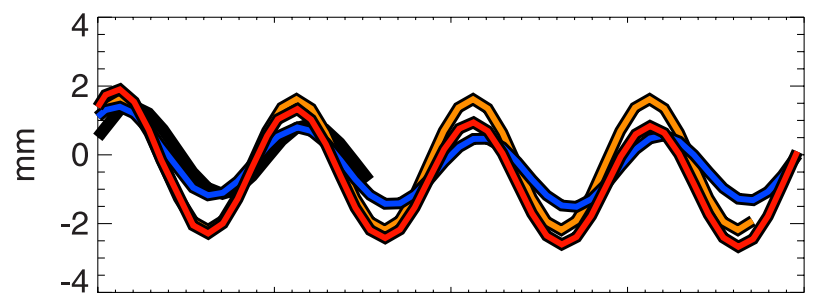

S_11

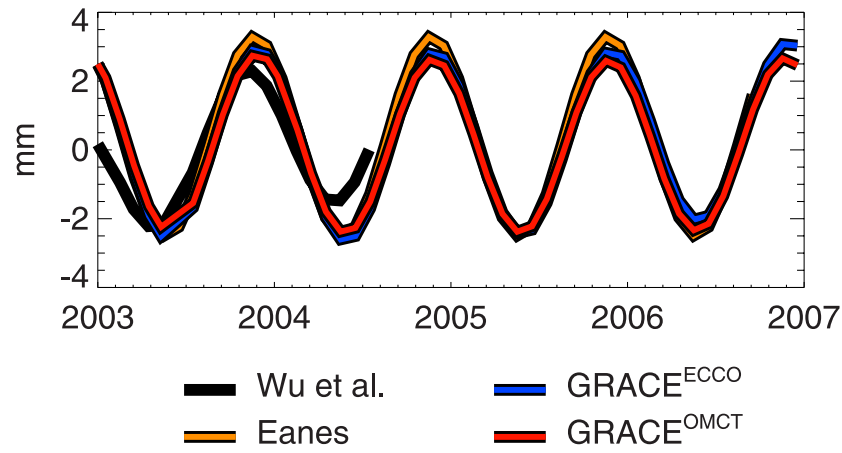

Figure 4. Same as Figure 3 except that monthly time series have been low-pass filtered.

ground stations [Wu et al., 2002], which are relatively sparsely distributed in the southern hemisphere.

\subsection{GRACE GSM Degree One Estimates}

[44] The GSM coefficients produced by the GRACE Project have had modeled estimates of the oceanic and atmospheric components of the gravity field removed. To provide a consistent set of degree one coefficients for

Table 1. Mean Annual Full Geocenter Variations

\begin{tabular}{|c|c|c|c|c|c|c|}
\hline & $X$ & $\sigma_{X}$ & $Y$ & $\sigma_{Y}$ & $Z$ & $\sigma_{Z}$ \\
\hline \multicolumn{7}{|c|}{ Mean Annual Amplitude [mm] } \\
\hline $\mathrm{GRACE}^{E C C O}$ & 1.13 & 0.07 & 2.67 & 0.09 & 1.23 & \\
\hline GRACE $^{O M C T}$ & 1.88 & 0.07 & 2.59 & 0.09 & 1.78 & 0.1 \\
\hline Chen et al. [1999] & 2.2 & & 3.2 & & 2.8 & \\
\hline Eanes [2000] & 1.9 & & 2.9 & & 2.3 & \\
\hline Cretaux et al. [2002] & 2.6 & 0.5 & 2.5 & 0.1 & 3.3 & 1.0 \\
\hline Wu et al. [2006] & 1.8 & 0.4 & 2.5 & 0.3 & 3.9 & 0.4 \\
\hline \multicolumn{7}{|c|}{ Mean Annual Phase [days] } \\
\hline GRACE $^{E C C O}$ & 52 & 4 & -35 & 2 & 55 & 3 \\
\hline $\mathrm{GRACE}^{O M C T}$ & 46 & 4 & -34 & 2 & 60 & 5 \\
\hline Chen et al. [199 & 59 & & -66 & & 45 & \\
\hline Eanes $[2000]$ & 44 & & -40 & & 41 & \\
\hline Cretaux et al. [2002] & 32 & 7 & -57 & 4 & 36 & 10 \\
\hline Wu et al. [2006] & 46 & 15 & -36 & 5 & 28 & 5 \\
\hline
\end{tabular}

Table 2. Mean Annual GSM-like Geocenter Variations

\begin{tabular}{lcccccc}
\hline & $X$ & $\sigma_{X}$ & $Y$ & $\sigma_{Y}$ & $Z$ & $\sigma_{Z}$ \\
\hline Mean Annual Amplitude [mm] & & & & & & \\
$\quad$ GRACE & 0.83 & 0.07 & 1.39 & 0.09 & 1.25 & 0.10 \\
GRACE $^{\text {OMCT }}$ & 1.10 & 0.07 & 1.32 & 0.09 & 1.80 & 0.10 \\
Mean Annual Phase [days] $_{\text {GRACE }}^{E C C O}$ & 131 & 4 & -71 & 5 & 85 & 5 \\
GRACE $^{\text {OMCT }}$ & 86 & 4 & -73 & 5 & 80 & 5 \\
\hline
\end{tabular}

GRACE users, we estimate a set of "GSM-like" degree one coefficients as described above. Figure 5 shows our monthly GSM degree one coefficients, while Figure 6 shows the low-pass filtered time series. In both plots, we have converted our results into geocenter coordinates using (5), to help compare with other results. Although the GSMlike coefficients do not represent the same quantity as the full geocenter motions previously described, we plot them with the Wu et al. [2006] and Eanes [2000] results to give an indication of the relative contribution of the removed ocean/atmosphere signal, and to aid the visual comparison to Figures 3 and 4.
CSR RL04
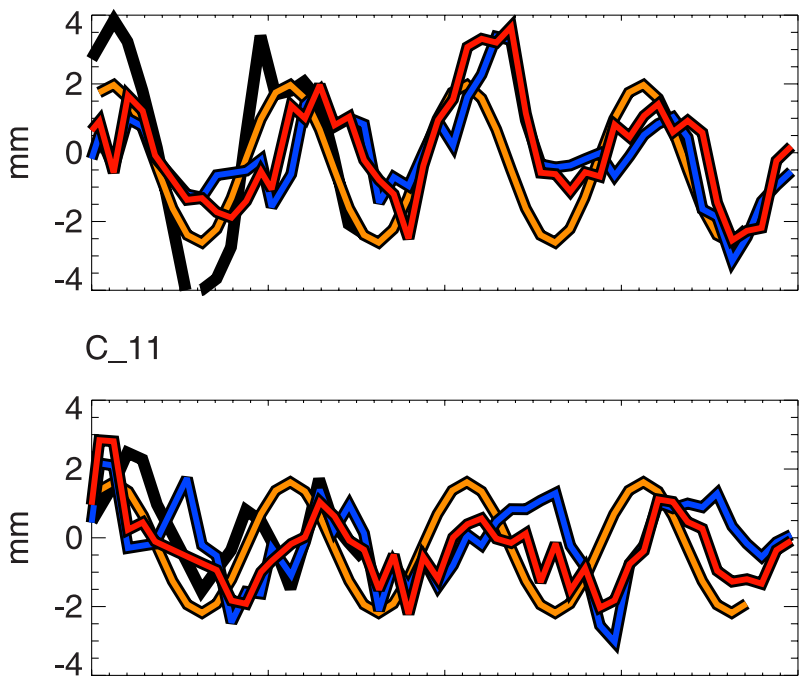

S_11

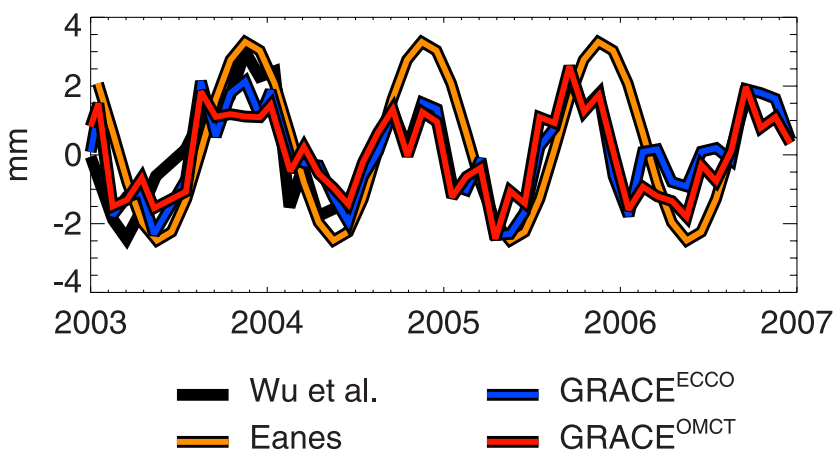

Figure 5. Same as Figure 3 except that here the monthly geocenter time series is GSM-like, in that it has had atmospheric and oceanic contributions removed corresponding to GRACE Level-2 data processing standard. 


\section{C_10}

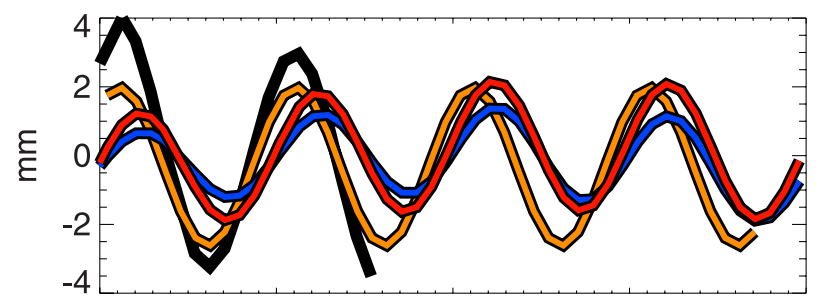

C_11

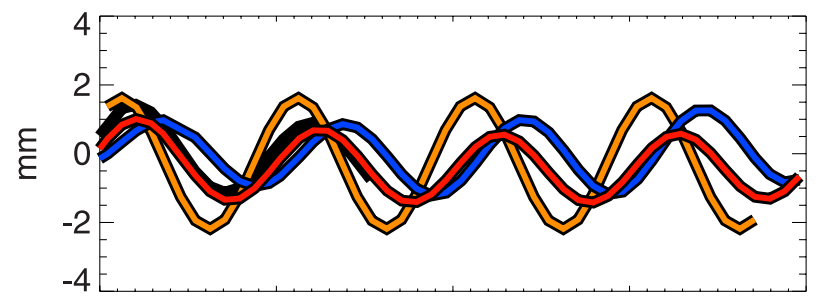

S_11

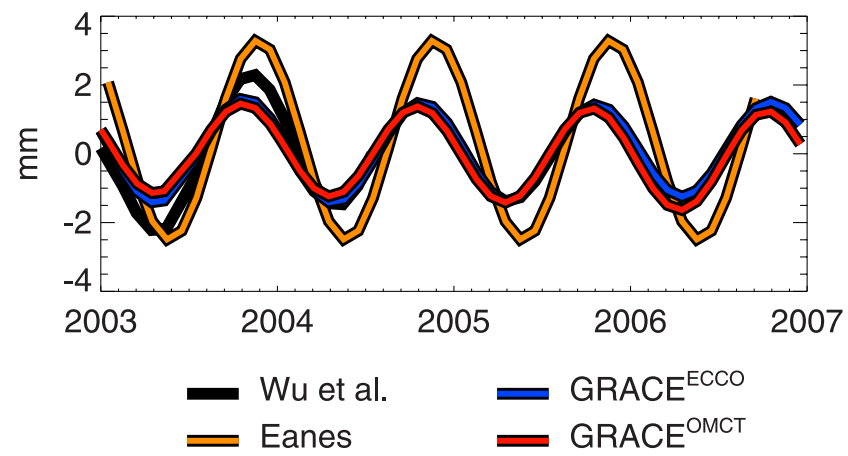

Figure 6. Same as Figure 5 except that monthly time series have been low-pass filtered.

[45] Because OMCT is used as the background ocean model during GRACE processing, the GRACE ${ }^{O M C T}$ GSMlike solution contains oceanic contributions from only the eustatic correction, the while GRACE ${ }^{E C C O}$ GSM-like solution includes the residual (ECCO minus OMCT) degree one ocean component in addition to the eustatic correction (see equation (16)). The modeled atmospheric and oceanic mass signals, which are not present in GSM-like solutions, contribute significantly to the full geocenter variability, as can be seen by comparing Figures 4 and 6 or the mean annual amplitudes and phases shown in Tables 1 and 2. The GRACE $^{E C C O}$ GSM-like values of $X, Y$, and $Z$ are $73 \%, 52 \%$, $101 \%$ of their full geocenter values, while GRACE ${ }^{O M C T}$ GSM-like values are $59 \%, 51 \%$, and $101 \%$ of their respective full geocenter values. The $Y$ GSM-like coefficients show the largest change in amplitude relative to their full geocenter values for both GRACE ${ }^{E C C O}$ and GRACE ${ }^{O M C T}$, and the GRACE ${ }^{O M C T} X$ also changes significantly. The annual phases are shifted by 1-2 months. The GSM-like $Z$ coefficients are similar to their full geocenter values, with slightly larger amplitudes and phases delayed by about one month.

[46] An advantage of this method relative to GPS- and SLR-based solutions is its ability to recover trends in the degree one coefficients. GPS inversions are predicated on an elastic model of Earth deformation; viscoelastic motions such as post-glacial rebound will therefore lead to mismodeled long period mass loads. To avoid these effects, $W u$ et al. [2006] detrend all GPS and OBP observations prior to inversion. SLR solutions are also typically detrended because trends in geocenter motion are indistinguishable from drift in the reference frame used in the solution. The trends in our solutions are presented in Table 3 . The trends for both solutions are sensitive to the time period used in the analysis, indicating that a longer time series is needed to derive significant values.

\subsection{Uncertainty Estimates}

[47] The synthetic experiment of section 6.1 demonstrated the viability of this method in the absence of errors other than truncation. Additional potential sources of error in this method are the output of the ocean models and the errors in the GRACE Stokes coefficients. The uncertainty in the modeled OBP estimates is unknown, but the difference between the GRACE ${ }^{O M C T}$ and GRACE ${ }^{E C C O}$ time series gives a qualitative idea of the amplitude of these errors. We assess the errors in the GRACE data following Wahr et al. [2004], which uses the root variance about the bestfitting annual cycle for each coefficient as a conservative error estimate. Here we modify this approach by using the variation about a low-pass filtered time series, rather than about the annual cycle. Using this error estimate, we find uncertainties in $G_{10 C}, G_{11 C}$, and $G_{11 S}$ of $0.22,0.23$, and $0.24 \mathrm{~mm}$ respectively. Propagating these uncertainties result in errors in the monthly values of $0.49,0.34,0.43 \mathrm{~mm}$ for $Z$, $X$, and $Y$.

\subsection{GRACE ${ }^{\text {OMCT }}$ and SLR Comparison}

[48] The studies of Chambers et al. [2004] and Chambers [2006] examined the contribution of degree one terms in GRACE estimates of mean sea level and steric sea level, and found substantial improvement in their results by including the SLR mean annual geocenter estimate of Eanes [2000]. Here we compare the relative impacts of the GRACE $^{O M C T}$ and SLR geocenter estimates on GRACE estimates of ocean bottom pressure.

[49] Figure 7 (top) shows the root variance of the difference between OBP estimates derived from GRACE and the ECCO ocean model. Both fields are smoothed with a $500 \mathrm{~km}$ Gaussian filter, and converted to $\mathrm{cm}$ of water thickness. The GRACE result includes the SLR mean annual geocenter estimate. The largest RMS differences can be seen in the North Atlantic, North Pacific, Indian Ocean, and the Southern Ocean near West Antarctica. Figure 7 (bottom) shows the improvement in the comparison if the GRACE ${ }^{O M C T}$ geocenter solution is used instead of the SLR solution. The RMS

Table 3. Geocenter Trends $[\mathrm{mm} / \mathrm{yr}]$

\begin{tabular}{lcccccc}
\hline & $X$ & $\sigma_{X}$ & $Y$ & $\sigma_{Y}$ & $Z$ & $\sigma_{Z}$ \\
\hline Full Coefficients & & & & & & \\
GRACE $^{\text {GCCO }}$ & -0.12 & 0.04 & 0.07 & 0.03 & -0.14 & 0.05 \\
GRACE $^{\text {OMCT }}$ & -0.20 & 0.04 & -0.02 & 0.03 & 0.06 & 0.05 \\
GSM Coefficients & & & & & & \\
GRACE $^{E C C O}$ & 0.06 & 0.04 & 0.04 & 0.03 & -0.06 & 0.05 \\
GRACE $^{\text {OMCT }}$ & -0.02 & 0.04 & 0.05 & 0.03 & 0.14 & 0.05 \\
\hline
\end{tabular}



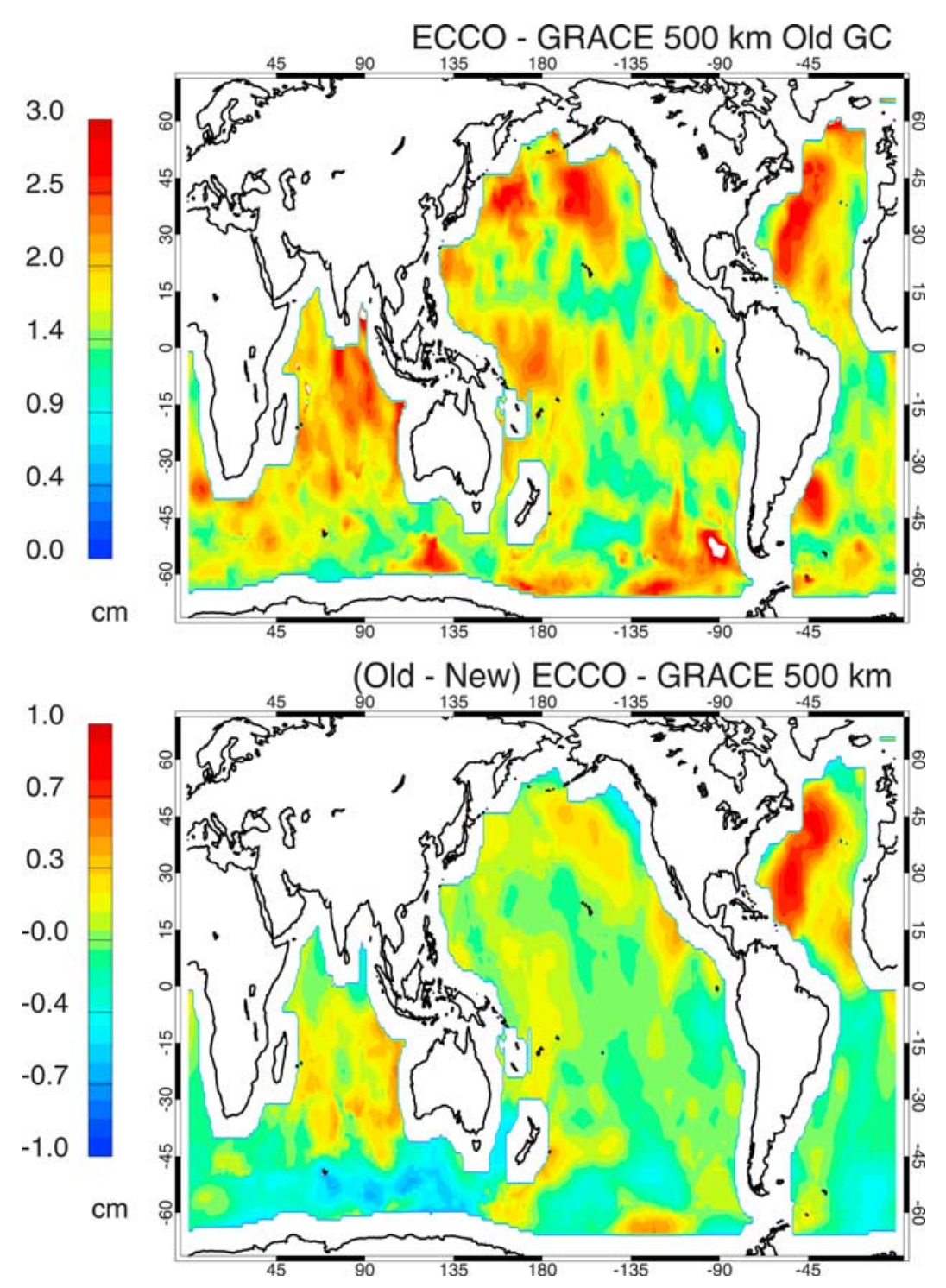

Figure 7. (top) RMS difference of GRACE and ECCO ocean bottom pressure estimates, smoothed with a 500-km Gaussian filter, in centimeters of equivalent water thickness. The GRACE estimate includes the SLR-based seasonal degree one coefficients. (bottom) Difference in RMS difference from using GRACE ${ }^{O M C T}$ monthly degree one coefficients.

difference is reduced by more than $1 \mathrm{~cm}$ in the North Atlantic, by about $0.5 \mathrm{~cm}$ in the North Pacific and Indian Ocean, and increased by about $0.5 \mathrm{~cm}$ in the Southern Ocean.

\section{Summary}

[50] In this study we have outlined a method for estimating the Earth's geocenter motion by combining GRACE data with a modeled ocean component of geocenter. Advantages of this algorithm are its simplicity, and its use of publicly available data (GSM and GAC files) provided by the GRACE Project. A GRACE user can improve his or her estimates of mass variability by filling the degree one data gap with a reliable, up-to-date estimate, that is consistent with the other GRACE GSM coefficients.

[51] At first glance, it may seem counter-intuitive to use GRACE data to solve for geocenter motion, given that GRACE is inherently insensitive to these terms. To under- stand the method described by equation (12), suppose all Stokes coefficients are zero except for $C_{10}^{\prime}$. We would then know that the global mass distribution is completely described by $A \sin \left(\right.$ latitude), because $P_{10}$ is proportional to $\sin$ (latitude). The spatial pattern is known, but $A$ is unknown. However, we can determine the amplitude, $A$, if we're also given the mass variation in some region, in this case the ocean. The purpose of the denominator of the right-hand side of equation (11) is to fix the amplitude $A$ from a $P_{10}$ mass distribution concentrated over that region.

[52] The application of the method on synthetic data shows that the unknown geocenter values can be reproduced given knowledge of the contribution of ocean bottom pressure and the higher degrees of the gravity field. In the presence of errors in the GRACE data, the monthly solutions have uncertainties in the range of 0.3 to $0.4 \mathrm{~mm}$. Mean annual estimates have uncertainties of $<0.1 \mathrm{~mm}$. The accuracy of the ocean models is unknown, but the differ- 


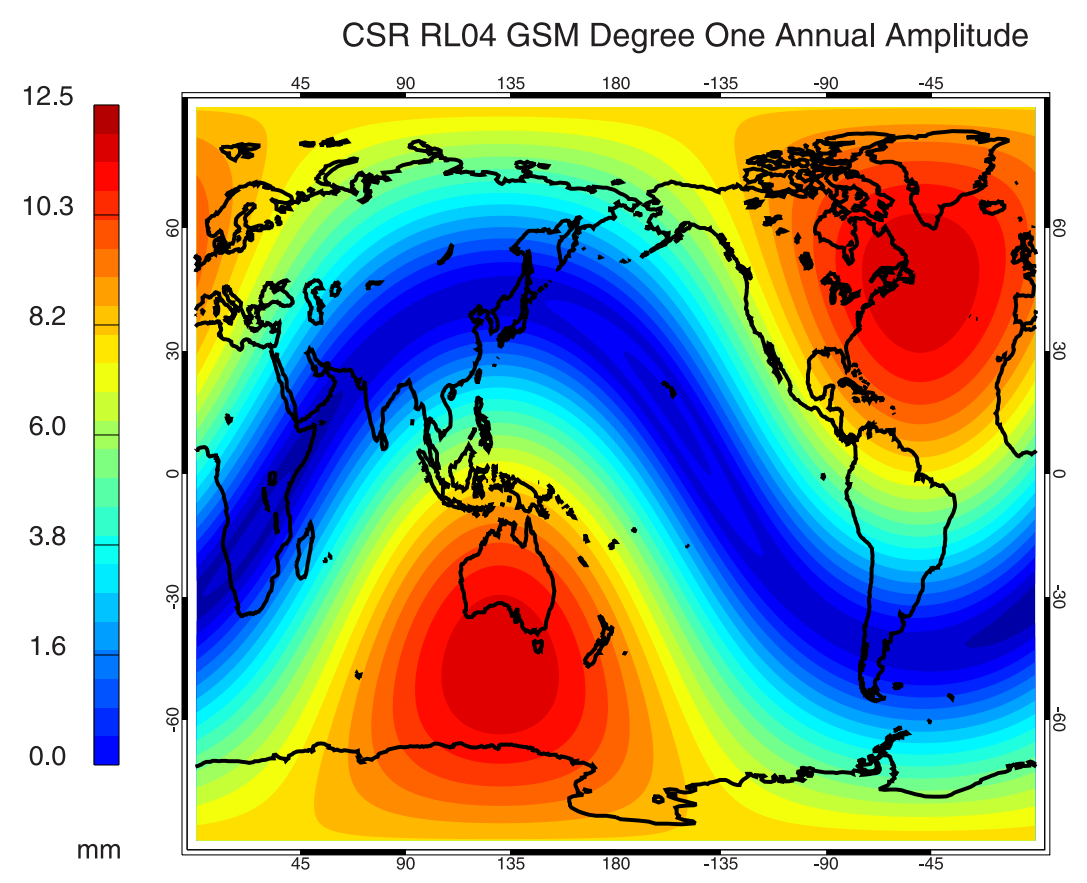

Figure 8. Annual amplitude of degree one contribution to surface mass estimates, based on OMCT ocean model, in millimeters of equivalent water thickness.

ences between the two models used here indicate these errors may be the limiting error source. We also demonstrate how this method may be altered to produce coefficients that are consistent with the standard processed GRACE GSM gravity fields; because such coefficients are not produced by the GRACE Project, omission of these coefficients is currently a source of error in GRACE derived surface mass estimates.

[53] To assess the significance of the exclusion of degree one terms from surface mass estimates made using GRACE data (as is currently the case), we use the best-fitting annual amplitudes of our geocenter estimates to construct maps of the surface mass variation due solely to these degree one terms. Figure 8 shows the contribution to the annual amplitude of a surface mass estimate from degree one components, in units of equivalent water thickness.

[54] Because of the long wavelength of the degree one terms, these values will be little changed by spatial averaging, and so can be used to estimate the likely omission errors in Gaussian smoothed results at the typically used averaging radii of $\sim 500-1000 \mathrm{~km}$. The maximum errors shown in Figure 8 approach $14 \mathrm{~mm}$. The mean global ocean value is $0.9 \mathrm{~mm}$, or about $10 \%$ of the seasonal global mean sea level amplitude.

[55] While we have chosen to model the oceanic component of geocenter when deriving (12), the region of the Earth to be modeled is in principle arbitrary. For example, one could apply this technique using a land surface model to estimate the degree one contribution of the continental regions. This method may also be amenable to the incorporation of GPS data. Rather than attempting to use GPS data to invert for the global geocenter coefficients, one might estimate just a regional degree one component (e.g., for North America). In this case, the effects of the GRACE truncation would need to be studied, as higher degree coefficients are apt to be more important for smaller regions.

[56] Acknowledgments. We wish to thank Frank Wu for providing his geocenter estimates and Archie Paulson for providing post-glacialrebound coefficients. D. Chambers was supported by a grant from the NASA Energy- and Water Cycle Program via a subcontract from UC-Irvine. John Wahr was supported in part by a NASA REASoN CAN award (through a contract with the Jet Propulsion Laboratory). The National Center for Atmospheric Research is sponsored by the National Science Foundation. We wish to thank B. Vermeersen and an anonymous reviewer for their constructive comments which led to the improvement of the manuscript.

\section{References}

Argus, D. F., W. R. Peltier, and M. M. Watkins (1999), Glacial isostatic adjustment observed using very long baseline interferometry and satellite laser ranging geodesy, J. Geophys. Res., 104, 29,077-29,093.

Blewitt, G. (2003), Self-consistency in reference frames, geocenter definition, and surface loading of the solid Earth, J. Geophys. Res., 108(B2), 2103, doi:10.1029/2002JB002082.

Blewitt, G., and P. Clarke (2003), Inversion of Earth's changing shape to weigh sea level in static equilibrium with surface mass redistribution, J. Geophys. Res., 108(B6), 2311, doi:10.1029/2002JB002290.

Chambers, D. P. (2006), Observing seasonal steric sea level variations with GRACE and satellite altimetry, J. Geophys. Res., 111, C03010, doi:10.1029/2005JC002914.

Chambers, P. D., J. Wahr, and R. S. Nerem (2004), Preliminary observations of global ocean mass variations with GRACE, Geophys. Res. Lett., 31, L13310, doi:10.1029/2004GL020461.

Chambers, D., M. Tamisiea, R. S. Nerem, and J. Ries (2007), Effects of ice melting on GRACE observations of ocean mass trends, Geophys. Res. Lett., 34, L05610, doi:10.1029/2006GL029171.

Chen, J. L., C. R. Wilson, R. J. Eanes, and R. S. Nerem (1999), Geophysical interpretation of observed geocenter variations, J. Geophys. Res., 104, 2683-2690.

Chen, J. L., M. Rodell, C. R. Wilson, and J. S. Famiglietti (2005), Low degree spherical harmonic influences on Gravity Recovery and Climate Experiment (GRACE) water storage estimates, Geophys. Res. Lett., 32, L14405, doi:10.1029/2005GL022964.

Cheng, M., and B. D. Tapley (2004), Variations in the Earth's oblateness during the past 28 years, J. Geophys. Res., 109, B09402, doi:10.1029/ 2004JB003028. 
Cretaux, J.-F., L. Soudarin, F. J. M. Davidson, M.-C. Gennero, M. BergeNguyen, and A. Cazenave (2002), Seasonal and interannual geocenter motion from SLR and DORIS measurements: Comparison with surface loading data, J. Geophys. Res., 107(B12), 2374, doi:10.1029/ 2002JB001820

Crowley, J. W., J. X. Mitrovica, R. C. Bailey, M. E. Tamisiea, and J. L. Davis (2006), Land water storage within the Congo Basin inferred from GRACE satellite gravity data, Geophys. Res. Lett., 33, L19402, doi:10.1029/2006GL027070.

Davis, J. L., P. Elsegui, J. X. Mitrovica, and M. E. Tamisiea (2004), Climate-driven deformation of the solid Earth from GRACE and GPS, Geophys. Res. Lett., 31, L24605, doi:10.1029/2004GL021435.

Eanes, R. (2000), SLR Solutions from the University of Texas Center for Space Research, Geocenter from Topex SLR/DORIS, 1992-2000, archived at IERS Special Bureau for Gravity/Geocenter. (Available at http://sbgg.jpl.nasa.gov/datasets.html)

Flechtner, F. (2007), AOD1B Product Description Document for Product Releases 01 to 04, GRACE 327-750, CSR publ. GR-GFZ-AOD-0001 Rev. 3.1, University of Texas at Austin, 43 pp.

Fukumori, I., R. Raghunath, L. Fu, and Y. Chao (1999), Assimilation of TOPEX/POSEIDON data into a global ocean circulation model: How good are the results?, J. Geophys. Res., 107, 25,647-25,665.

Han, S. C., C. K. Shum, M. Bevis, and C. Y. Kuo (2006), Crustal dilatation observed by GRACE after the 2004 Sumatra-Andaman earthquake, Science, 313, 658-662.

Marshall, J., A. Adcroft, C. Hill, L. Perelman, and C. Heisey (1997), A finite-volume, incompressible Navier-Stokes model for studies of the ocean on parallel computers, J. Geophys. Res., 102, 5753-5766.

Paulson, A., S. Zhong, and J. Wahr (2007), Inference of mantle viscosity from GRACE and relative sea level data, Geophys. J. Int., 171(2), 497508.

Peltier, W. R. (2004), Global glacial isostasy and the surface of the Ice-Age Earth: The ICE-5G (VM2) model and GRACE, Annu. Rev. Earth Planet. Sci., 32, 111-149.

Rodell, M., et al. (2004), The global land data assimilation system, Bull. Am. Meteorol. Soc., 85, 381-394.

Schmidt, R. T., et al. (2006), GRACE observations of changes in continental water storage, Global Planet. Change, 50, 112-126.

Seo, K. W., C. R. Wilson, J. S. Famiglietti, J. L. Chen, and M. Rodel (2006), Terrestrial water mass load changes from gravity recovery and climate experiment (GRACE), Water Resour. Res., 42, W05417, doi:10.1029/2005WR004255

Swenson, S., J. Famiglietti, J. Basara, and J. Wahr (2008), Estimates of Groundwater Variability in Oklahoma from GRACE and in situ soil moisture data, Water Resour. Res., 44, W01413, doi:10.1029/ 2007WR006057.

Tamisiea, M. E., J. X. Mitrovica, G. A. Milne, and J. L. Davis (2001), Global geoid and sea level changes due to present-day ice mass fluctuations, J. Geophys. Res., 106, 30,849-30,863.
Tamisiea, M. E., E. W. Leuliette, J. L. Davis, and J. X. Mitrovica (2005), Constraining hydrological and cryospheric mass flux in southeastern Alaska using space-based gravity measurements, Geophys. Res. Lett., 32, L20501 doi:10.1029/2005GL023961.

Tamisiea, M. E., J. X. Mitrovica, and J. L. Davis (2007), GRACE Gravity Data Constrain Ancient Ice Geometries and Continental Dynamics over Laurentia, Science, 316, 881-883.

Tapley, B. D., S. Bettadpur, M. Watkins, and Ch. Reigber (2004a), The gravity recovery and climate experiment: Mission overview and early results, Geophys. Res. Lett., 31, L09607, doi:10.1029/2004GL019920.

Tapley, B. D., S. Bettadpur, J. C. Ries, P. F. Thompson, and M. M. Watkins (2004b), GRACE measurements of mass variability in the Earth system, Science, 305, 503-505

Thomas, M. (2002), Ocean induced variations of Earth's rotation-Results from a simultaneous model of global circulation and tides, Ph.D. dissertation, 129 pp., Univ. of Hamburg, Germany.

Trupin, A. S., M. F. Meier, and J. M. Wahr (1992), The effect of melting glaciers on the Earth's rotation and gravitational field: 1965-1984, Geophys. J. Int., 108, 1-15.

Velicogna, I., and J. Wahr (2006), Measurements of time-variable gravity show mass loss in Antarctica, Science, 311, 1754-1756.

Wahr, J., M. Molenaar, and F. Bryan (1998), Time variability of the Earth's gravity field: Hydrological and oceanic effects and their possible detection using GRACE, J. Geophys. Res., 103(B12), 30,205-30,229.

Wahr, J., S. Swenson, V. Zlotnicki, and I. Velicogna (2004), Time-Variable Gravity from GRACE: First Results, Geophys. Res. Lett., 31, L11501, doi:10.1029/2004GL019779.

Wu, X., D. F. Argus, M. B. Heflin, E. R. Ivins, and F. H. Webb (2002), Site distribution and aliasing effects in the inversion for load coefficients and geocenter motion from GPS data, Geophys. Res. Lett., 29(24), 2210 , doi:10.1029/2002GL016324.

Wu, X., M. B. Heflin, E. R. Ivins, and I. Fukumori (2006), Seasonal and interannual global surface mass variations from multisatellite geodetic data, J. Geophys. Res., 111, B09401, doi:10.1029/2005JB004100.

Yeh, P. J.-F., S. C. Swenson, J. S. Famiglietti, and M. Rodell (2006), Remote sensing of groundwater storage changes in Illinois using the Gravity Recovery and Climate Experiment (GRACE), Water Resour Res., 42, W12203, doi:10.1029/2006WR005374.

D. Chambers, Center for Space Research, University of Texas, 3925 W. Braker Lane, Suite 200, Austin, TX 78759, USA.

S. Swenson, Advanced Study Program, National Center for Atmospheric Research, P.O. Box 3000, Boulder, CO 80307, USA. (swensosc@ucar.edu)

J. Wahr, Department of Physics and Cooperative Institute for Research in Environmental Sciences, University of Colorado, CB390, Boulder, CO 80309, USA. 